\title{
Diffuse pulmonary ossification in a patient exposed to
}

silica

\section{To the Editor:}

Pulmonary ossification is a rare disease characterised by the formation of diffuse small fragments of mature bone tissue in the lungs. It can be idiopathic or associated with underlying chronic lung, heart or systemic disorders. We present the case of an 83-year-old male, who had diffuse dendriform pulmonary ossification (DPO) and a spontaneous pneumothorax.

An 83-year-old male nonsmoker with a history of hypertension and carotid and coronary angioplasties visited his medical practitioner for right chest pain after a flight from Spain to France. He had no history of lung disease or heart failure.

He worked as a dentist with a dental prosthesis manufacturer. Our patient was frequently in the same workshop as the prostheses technician.

The patient had no trauma, cough, sputum disorders or dyspnoea. A physical examination and the usual biological analyses were normal, particularly the phosphocalcic values.

Chest radiography revealed a partial right pneumothorax with micronodular calcified opacities in both lungs. Computed tomography revealed the presence of bilateral disseminated micronodular opacities of calcified densities, predominantly located in the lower lung, which had lower densities than silicosis. There were no calcified lymph nodes in the mediastinum (figs 1 and 2).

Despite drainage, because the pneumothorax was persistent, the patient underwent video-assisted thoracoscopic surgery 2 days later. The lung was particularly "rigid" and lamellar deposits of calcified osteoid material, with marrow elements in the alveolar spaces and interstitium, were found in the lung biopsies (fig. 3). The lung parenchyma was otherwise normal. There was no suggestion of any pneumoconiosis, fibrosis or inflammation, and no evidence of venous congestion or iron deposition. The final diagnosis was DPO.

The patient's recovery was favourable, and lung function tests and echocardiography were normal a few months after surgery.

Pulmonary ossification is characterised by the presence of mature bone in alveolar or interstitial spaces, either localised or disseminated throughout the lung parenchyma. It has no specific clinical features, but two histological types are described: DPO and nodular pulmonary ossification (NPO).

DPO is characterised by linear and branching bone deposits within the interstitium. It may include bone marrow, fat and hematopoietic cells. Associations with chronic obstructive pulmonary disease, idiopathic pulmonary fibrosis, asbestosis, amyloidosis, busulfan therapy and pneumoconiosis after inhalation of rare earth metals have been described [1]. Although fatal DPO with almost total ossification of the lungs has been described, it usually remains a benign disorder, commonly found in males aged >60 years. Radiologically, DPO is characterised by branching linear shadows of calcified

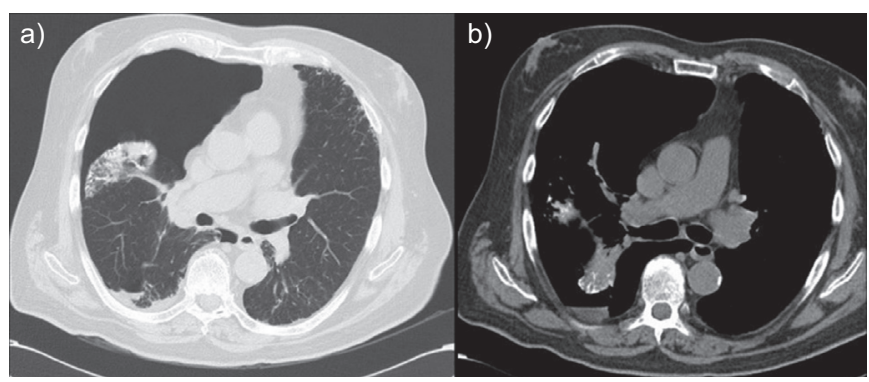

FIGURE 1. a) Computed tomography (CT) of the chest in a parenchymal window demonstrating a right pneumothorax and a nodular round shadow in the collapsed lung; b) CT of the chest in a mediastinal window demonstrating micronodular calcified opacities.

densities extending along the bronchovascular distribution of the lower lungs. It is often interpreted as pulmonary fibrosis, bronchiectasis or lymphangitis [1].

The main differential diagnoses of DPO are silicosis, disseminated dendriform pulmonary ossification associated with usual interstitial pneumonia (UIP), pulmonary alveolar microlithiasis (PAM) and chicken pox. Nodular calcifications can also be found in other diseases like tuberculosis, metastatic osteosarcoma, amyloidosis and silicoproteinosis. Density is less important in DPO than in silicosis and there are no calcified lymph nodes in the mediastinum. In DPO/UIP, there are fewer calcifications and these are smaller in size $(0.5-3 \mathrm{~mm}$ in diameter) than those expected from multiple ossifications. In DPO/UIP, with thin-section computed tomography (osteoporosis window), DPO is detected as multiple tiny calcifications in bibasilar subpleural lungs, where characteristics features suggestive of UIP (honeycomb cystic changes, irregular linear opacities and traction bronchiectasis) are predominant [2]. Finally, PAM is characterised by deposition in the alveoli of spherical calcium phosphate concretions (microliths or
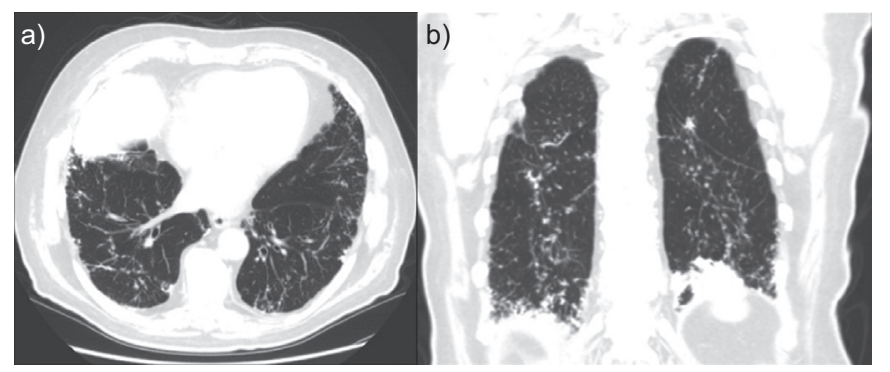

FIGURE 2. a) Computed tomography (CT) at 8 months after surgery demonstrating an irregular distribution of multiple micronodular calcified opacities particularly in the subpleural areas and lower lobes; b) coronal-reformatted CT image of the chest 8 months after surgery demonstrating an irregular distribution of multiple nodular calcified opacities in the full height of the lungs. 


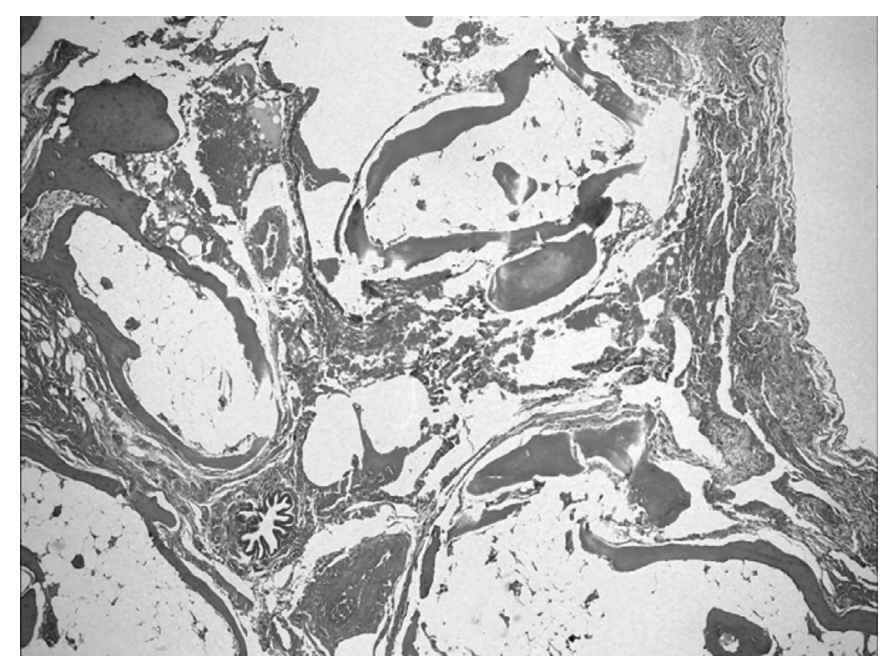

FIGURE 3. Diffuse bony deposits in interstitium and alveolar lumina, some containing marrow elements composed of mature fat and haematopoietic cells.

calcipherites) without bone marrow [3]. In this disease, patients may remain asymptomatic for many years but do usually become symptomatic between the third and fourth decades [4]. In some patients, it progresses into pulmonary fibrosis, respiratory failure and cor pulmonale. Typical complaints are dyspnoea, nonproductive cough, chest pain and asthenia [3, 4]. High attenuation of lung parenchyma caused by calcipherites, even smaller than $1 \mathrm{~mm}$, is visible on high-resolution computed tomography (HRCT) with a gradient of distribution of the calcifications, in which the lung bases, especially the posterior, are more affected than the middle or upper zones, with a greater concentration in the subpleural parenchyma and along the bronchovascular bundles, whereas HRCT shows thickening of the lobular septa with a distribution of microliths along the septa and around the centrilobular distal bronchioles [5]. In PAM, extrapulmonary calcifications, mainly in the urinary tract, are typically described [3].

NPO tends to be more circumscribed than DPO, DPO/UIP or PAM, with ossific bodies located in the alveolar space without a marrow component. It is usually associated with chronic heart disorders, such as mitral stenosis [6], idiopathic hypertrophic subaortic stenosis and pulmonary oedema [7]. Thus, NPO may develop from pulmonary congestion-inducing disorders. Radiologically, it manifests as scattered subpleural nodular calcified densities ( $<1 \mathrm{~cm}$ diameter), located in the lower lungs.

Our patient suffered from mild atheromatosis but had no mitral valve disease, subaortic stenosis, or history of rheumatic fever, recurrent pulmonary oedema, or heart or respiratory failure. Although he was exposed to silica, this radiological presentation was not typical of silicosis and the biopsies were not compatible with that diagnosis. No mineralogical analysis was made on the biopsies.

To our knowledge, two other cases of DPO have been reported with spontaneous pneumothorax $[8,9]$. One case occurred in a pilot [7] and our patient also had a pneumothorax following air travel.
Our patient was also regularly exposed to silica during the manufacture of dental prostheses. Could this occupational exposure be responsible for DPO?

DPO is a rare, often asymptomatic disease of unknown pathogenesis. Two histological types have been described: DPO and NPO. In our patient, DPO was discovered through a spontaneous pneumothorax a few days after air travel. Our patient's clinical and radiological presentation could have wrongly suggested the presence of silicosis, even though the computed tomography signs were atypical. Occupational exposure to silica may have played a role in the pathogenesis of this disease.

\section{Helene Jungmann*, Benoît Godbert", Marie-Pierre Wissler", Denis Regent ${ }^{+}$, Jean-Michel Vignaud", Zola Bavelele* and Yves Martinet ${ }^{\#}$}

* Service de médecine A, Centre hospitalier de Remiremont, Remiremont, " $\mathrm{CHU}$ de Nancy, Pôle de spécialités médicales, Service de pneumologie, Nancy, "CHU de Nancy, Pôle laboratoires, Service d'anatomo-pathologie, Nancy, and ${ }^{+} \mathrm{CHU}$ de Nancy, Pôle Radiologie, Service de radiologie, Nancy, France.

Correspondence: B. Godbert, Pôle de Spécialités médicales, Service de pneumologie, Bâtiment P. Canton, Hôpitaux de Brabois, Rue du Morvan, F-54500 Vandoeuvre les Nancy, France. E-mail: benoit.godbert@hp-metz.fr

Statement of Interest: Conflict of interest information can be found alongside the online version of this article at err. ersjournals.com

Provenance: Submitted article, peer reviewed.

\section{REFERENCES}

1 Chan ED, Morales DV, Welsh CH, et al. Calcium deposition with or without bone formation in the lung. State of the Art. Am J Respir Crit Care Med 2002; 165: 1654-1669.

2 Kim TS, Han J, Chung MP, et al. Disseminated dendriform pulmonary ossification associated with usual interstitial pneumonia: incidence and thin-section CT-pathologic correlation. Eur Radiol 2005; 15: 1581-1585.

3 Jönsson ALM, Simonsen U, Hilberg O, et al. Pulmonary alveolar microlithias: two case reports and review of literature. Eur Respir Rev 2012; 21: 249-256.

4 Abdalla G, Marchiori E, Zanetti G, et al. Pulmonary alveolitis microlithiasis: a case report with emphasison imaging findings. Case Report Med 2010; 2010: 819242.

5 Siddiqui NA, Fuhrman CR. Best cases from the AFIP: pulmonary alveolar microlithiasis. Radiographics 2011; 31: 585-590.

6 Galloway RW, Epstein EJ, Coulshed N. Pulmonary ossific nodules in mitral valve disease. Br Heart J 1961; 23: 297-307.

7 Popelka CG, Kleinerman J. Diffuse pulmonary ossification. Arch Intern Med 1977; 137: 523-525.

8 Ekhold PF, Oppedal BR, Arva P. Diffuse pulmonary ossification and spontaneous pneumothorax in a pilot: a case report. Aviat Space Environ Med 1986; 57: 696-698.

9 Ikeda Y, Yamashita H, Tamura T. Diffuse pulmonary ossification and recurrent pneumothorax in a patient with bronchial asthma. Respir Med 1998; 92: 887-889. 